\title{
Transporte de oxígeno por plantas macrófitas en aguas residuales de origen acuícola
}

\author{
Oxygen transport by aquatic macrophytes in wastewater
}

\author{
Cristóbal Medina ${ }^{1}$, Ernesto Tunnerman ${ }^{2}$, Eli Coleman ${ }^{3}$, Kenneth Fonseca $^{3}$ \\ ${ }_{1}^{1}$ MSc. Ciencias Ambientales, ORCID : https://orcid.org/0000-0001-9848-3589/ cmedina@ci.una.edu.ni \\ ${ }^{2}$ Ingeniero Químico, ORCID: https://orcid.org/0000-0001-8904-6969 / etunnermann@ci.una.edu.ni \\ ${ }^{3}$ Graduados de la carrera de Ingeniería en Recursos Naturales, ORCID: https://orcid.org/0000-0002-9803-2603 / \\ elicolemanbarrow@gmail.com / ORCID: https://orcid.org/0000-0001-7885-0935 / \\ Wolfalex350gmail.com \\ Facultad de Recursos Naturales y del Ambiente, Universidad Nacional Agraria
}

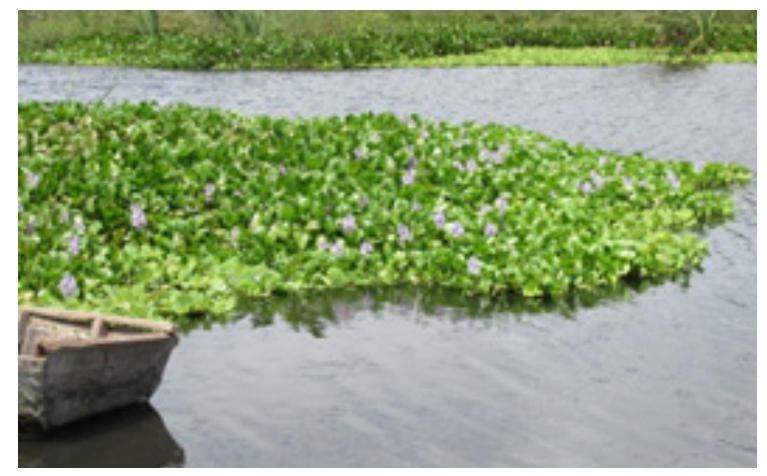

\section{RESUMEN}

Las macrófitas transportan oxigeno atmosférico a la rizosfera creando un ambiente aireados para los miroorganismos que contribuyen a la disminución de la demanda biológica de oxígeno, demanda química de oxígenos y sólidos suspendido, así como en la remoción de sustancias orgánicas como nutrientes, metales pesados, fenoles, ácido fórmico, colorantes, pesticidas entre otros. Esta investigación pretende evaluar a nivel de laboratorio el mejoramiento de la calidad de aguas residuales utilizando plantas acuáticas de jacinto (Eichhornia crassipes) y lechuga de agua (Pistia stratiotes). Los tratamientos evaluados fueron: plantas con barrera, plantas sin barrera, sin plantas con barrera y sin plantas sin barreras. El análisis de varianza al contenido de oxígeno disuelto en aguas residuales mostró diferencia significativa $(p<0.05)$ entre especies y tratamiento. Jacinto presenta valores promedios de 2.60 a $5.08 \mathrm{mg} \mathrm{l}^{-1}$ en un periodo de nueve días de observación, asimismo el tratamiento sin barrera muestra mayor valor en un rango de 2.7 a $4.85 \mathrm{mg} \mathrm{l}^{-1 .}$ Con lechuga de agua se observan mayores valores en el tratamiento sin barrera $\left(2.27\right.$ a $\left.4.81 \mathrm{mg}^{1-1}\right)$ y con barrera (1.24 a $\left.1.53 \mathrm{mg} \mathrm{l}^{-1}\right)$. Jacinto con barrera y sin barrera presenta valores de oxígeno disuelto transferido entre 0.46 y $0.45 \mathrm{mg} \mathrm{O}_{2} \mathrm{~d}^{-1} \mathrm{l}^{-1}$ respectivamente. Jacinto sin barrera removió en aguas residuales el $20.23 \%$ de la demanda biológica de oxígeno y con berrera el $19 \%$ al final del noveno día de observación. El tratamiento sin plantas sin barrera removió un promedio de $63 \%$, de la demanda biológica de oxígeno, mientras el tratamiento sin plantas con barrera fue de 42 $\%$. Jacinto sin barrera mostró mayor promedio de peso de biomasa
ABSTRACT
The macrophytas transports atmospheric oxygen to the rhizofera creating airy environments for the micro-organisms that contribute to the reduction of biological oxygen demand, chemical oxygen demand and suspended solids, also in the removal of organic substances such as nutrients, heavy metals, phenols, formic acid, colorants, pesticides among others. This research aims to evaluate the improvement of wastewater quality in the laboratory, using aquatic plant species of jacinto (Eichhornia crassipes) and water lettuce (Pistia stratiotes). The treatments evaluated were: Plants with Barriers, Plants without Barriers, Barriers without plants and without barriers nor plants. The content of dissolved oxygen in wastewater analysis of variance showed significant difference $(p<$ 0.05 ) between species and treatment. Jacinto had average values of 2.60 to $5.08 \mathrm{mg} \mathrm{l}^{-1}$ in a nine-day observation period, also the without Barriers treatment shows a higher value from 2.7 to $4.85 \mathrm{mg} \mathrm{l}^{-1}$. On the other hand, water lettuce showed higher values in the without Barriers treatment, ranging between 2.27 and $4.81 \mathrm{mg} \mathrm{l}^{-1}$ and with Barriers from 1.24 to $1.53 \mathrm{mg} \mathrm{l}^{-1}$. Jacinto Plants with Barriers and Plants without Barriers showed transferred dissolved oxygen values of 0.46 and $0.45 \mathrm{mg} \mathrm{O}_{2} \mathrm{~d}^{-1} \mathrm{l}^{-1}$ respectively. Jacinto without Barriers, removed the wastewater in a $20.23 \%$ of the biological oxygen demand and with Barriers $19 \%$ at the end of the ninth observation day. On the other hand, the without barriers nor plants treatment removed an average of $63 \%$ of the biological biochemical oxygen demand, while the Barriers without plants treatment was $42 \%$. Jacinto without Barriers showed higher average of total dry biomass
Recibido: 1 de abril del 2020

Aceptado: 4 de febrero del 2021

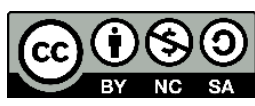

Los artículos de la revista La Calera de la Universidad Nacional Agraria, Nicaragua, se comparten bajo términos de la licencia Creative Commons: Reconocimiento, No Comercial, Compartir Igual. Las autorizaciones adicionales a las aquí delimitadas se pueden obtener en el correo edgardo.jimenez@ci.una.edu.ni

C) Copyright 2021. Universidad Nacional Agraria (UNA). 
seca total $(6.06 \mathrm{~g})$ y concentración de oxígeno disuelto $(3.63-5.34$ $\left.\mathrm{mg}^{-1}\right)$ lechuga de agua sin barrera $(3.20 \mathrm{~g})$ y oxígeno disuelto (1.7$\left.4.81 \mathrm{mg} \mathrm{l}^{-1}\right)$.

Palabras clave: plantas acuáticas, oxígeno disuelto, biomasa. weight $(6.06 \mathrm{~g})$ and dissolved oxygen concentration $(3.63-5.34 \mathrm{mg}$ $\left.\mathrm{1}^{-1}\right)$ compared with the water lettuce plant without Barriers (3.20 g) and dissolved oxygen $\left(1.7-4.81 \mathrm{mg}^{-1}\right)$.

Keywords: Aquatic plants, dissolved oxygen, biomass.

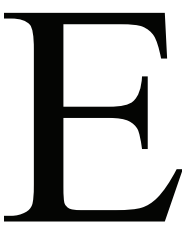

n Centroamérica existe un alto porcentaje de agua limpia que está siendo contaminada por su uso doméstico e industrial y vertidos a ríos y lagos sin recibir ningún tipo de tratamiento. Esta situación ha provocado que hoy en día la población se enfrente con graves problemas ambientales y afecciones de la salud vinculadas con aguas contaminadas, como son la generación de focos de vectores transmisores de enfermedades y la ingestión de alimentos contaminados por la irrigación de cultivos agrícolas con aguas residuales sin tratamiento (Arrianza et al, 2004, p. 9). Esto último acompañado con el cambio climático representa que el agua está menos disponible para el consumo humano, lo que hace necesario el mejoramiento de la calidad para reutilizarla, mediante alternativas menos costosas con el uso de plantas macrófitas, estas tienen como función airear el sistema radicular, facilitar oxígeno a los microorganismos que viven en la rizosfera, absorción de nutrientes (nitrógeno y fósforo), eliminación de contaminantes asimilándolos directamente en sus tejidos y filtración de los sólidos a través del entramado que forma su sistema radicular (Fernández, 2004, p. 79). Las plantas acuáticas han sido desarrolladas como un tratamiento secundario o terciario alternativo de aguas residuales, demostrando ser eficiente en la remoción de una amplia gama de sustancias orgánicas como nutrientes y metales pesados (Novotny y Olem, 1994, citado en Domínguez, 2003), la especie de jacinto de agua puede remover algunos compuestos orgánicos, como fenoles, ácido fórmico, colorantes, pesticidas, disminución de la demanda biológica de oxígeno (DBO), demanda química de oxígeno (DQO) y sólidos suspendidos (Metcalf y Eddy, 1995, citado en Domínguez, 2003).

En nuestro país, no se tiene evidencia de trabajos de investigación sobre la temática de plantas macrófitas en tratamiento de aguas residuales y aguas grises a nivel de laboratorio, sin embargo, existen experiencias relacionadas en la construcción de biofiltros que permite el uso de plantas naturales, para el tratamiento de las aguas residuales en poblaciones mayores de 3000 habitantes y en los hogares (biofiltro domiciliar), esta tecnología poco difundida ha presentado buenos resultados en cinco sitios en la zona del Pacífico de Nicaragua como Masaya, León, Managua, Chinandega y San Rafael del Sur (Schutze, 2010, p. 7). Con esta investigación se pretende incursionar en la temática de la biorremediación de las aguas residuales para evaluar dos especies de planta acuáticas en cuanto a transferencia de oxígeno disuelto para el mejoramiento de su calidad.

\section{MATERIALES Y MÉTODOS}

Este experimento, se realizó en el Laboratorios de Recursos Naturales de la Universidad Nacional Agraria (UNA), ubicado en el kilómetro 12 1/2 Carretera Norte, Managua, Nicaragua. Se colectaron plantas acuáticas flotantes de las especies Jacinto (Eichhornia crassipes) y Lechuga (Pistia stratiotes) en los lagos Xolotlán (Managua) y Apanás (Jinotega), luego se trasladó al Laboratorio de Calidad Ambiental. El agua residual utilizada fue obtenida de la granja demostrativa de peces de la UNA, la cual se introdujo en envase plástico de 1 $500 \mathrm{ml}$ envuelto en papel aluminio.

\section{Tratamientos}

Plantas con barrera (PCB). Planta de jacinto y/o lechuga de agua, en recipiente con agua residual con boca superior tapada con papel aluminio. PCB pretendió evaluar el transporte de $\mathrm{O}_{2}$ a través de la planta.

Planta sin barrera (PSB). Planta de jacinto y/o lechuga de agua, en recipiente con agua residual con boca superior descubierto. Este tratamiento permitió evaluar el transporte de $\mathrm{O}_{2}$ a través de la planta, así mismo en conjunto con la difusión simultánea de $\mathrm{O}_{2}$ en la columna de agua que rodea la planta.

Sin planta sin barrera (SPSB). Recipiente con agua residual, sin planta y boca superior descubierto. SPSB permitió la evaluación de la difusión del $\mathrm{O}_{2}$ de la atmósfera en la reducción de la DBO5 de las aguas residuales.

Sin planta con barrera (SPCB). Envase con aguas residuales y sellado herméticamente con tapón plástico. SPCB evalúa la función de los microorganismos anaeróbicos en la reducción de las $\mathrm{DBO}_{5}$ de las aguas residuales.

Cada tratamiento se constituyó de tres repeticiones, establecido en condiciones bajo luces con un ciclo de 12 horas y 12 horas oscuras, temperatura promedio de $25{ }^{\circ} \mathrm{C}$ durante nueve días de observación. Se registró diariamente datos de oxígeno disuelto (OD) con ayuda de un oxigenómetro marca OHAUS ST300D, se determinaron los pesos fresco y seco de la raíz y tallo de la planta. Al inicio y final del experimento se colectaron muestras de aguas residuales y se enviaron al Laboratorio del Centro de Investigación Acuática de la UNANManagua (CIRA/UNAN-Managua), para elanálisis de $\mathrm{DBO}_{5}$ por el método de Winkler, establecido en los métodos estándar. 
Análisis de los datos. Una vez recolectados los datos en el laboratorio se ordenaron por variable y por tratamiento para luego realizar un análisis de varianza (ANDEVA) utilizando el software estadístico InfoStat versión 2018, y se realizó separación de media por Tukey $(p=0.05)$.

\section{RESULTADOS Y DISCUSIÓN}

El contenido de OD en aguas residuales de origen acuícola mostró que jacinto, sin barrera (SB) presenta valores de 5.91 $\mathrm{mg}^{-1}$ al inicio del experimento, luego fluctúo en el rango de 3.64 a $5.37 \mathrm{mg} \mathrm{l}^{-1}$ OD hasta el final del noveno día de registro, superando al tratamiento con barrera (CB), que registró valores 1.42 a $4.81 \mathrm{mg} \mathrm{l}^{-1} \mathrm{OD}$ (Figura 1).

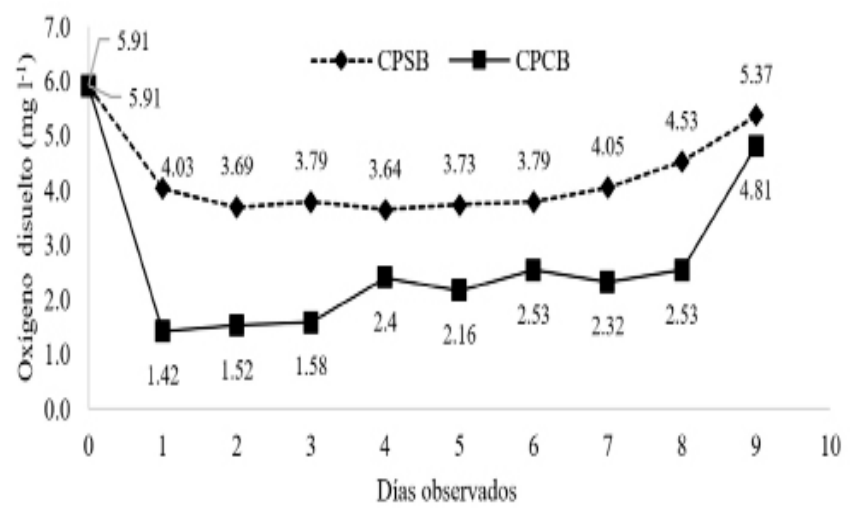

Figura 1. Oxígeno disuelto $\left(\mathrm{mg}^{1^{-1}}\right)$ en aguas residuales acuicola con planta Jacinto (Eichhornia crassipes) con y sin barrera. CPSB: Con plantas sin barrera; CPCB: Con plantas con barrera

Utilizando plantas de lechuga de agua $\mathrm{SB}$ se registran concentraciones de 1.68 a $4.35 \mathrm{mg} \mathrm{l}^{-1} \mathrm{OD}$, superando al tratamiento $\mathrm{CB}$, donde se observan valores de 1.01 a 1.60 $\mathrm{mg} \mathrm{l}^{-1} \mathrm{OD}$. El primer día de observación ambos tratamientos muestran un descenso de concentración de OD de $32 \%$ y 76 $\%$ respectivamente.

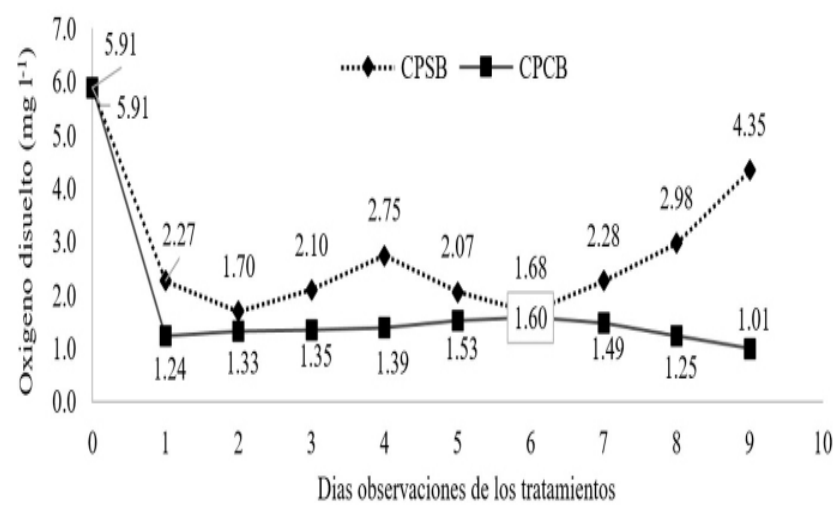

Figura 2. Oxígeno disuelto $\left(\mathrm{mg} \mathrm{l}^{-1}\right)$ en aguas residuales acuícola con planta de lechuga (Pistia stratiotes) con o sin barrera.

CPSB: Con plantas sin barrera; CPCB: Con plantas con barrera.
Se realizó análisis de varianza de concentración de OD en aguas residuales conteniendo plantas de jacinto y lechuga, mostrando diferencias significativas $(p<0.05)$ entre especie, tratamiento y periodo de observación. Jacinto mostró valores de 2.60 a $5.08 \mathrm{mg} \mathrm{l}^{-1}$, mientras, lechuga mostró valores menores de concentración de OD de $1.51 \mathrm{a} 2.75 \mathrm{mg}$ $1^{-1}$. En el periodo de observación jacinto mostró mayores concentraciones de OD comparado con lechuga (Cuadro 1).

Cuadro 1. Oxígeno disuelto (mg $\mathrm{l}^{-1}$ ) en aguas residuales de origen acuícola con plantas de jacinto y lechuga según días observados

\begin{tabular}{lccccccccc}
\hline \multirow{2}{*}{ Especie } & \multicolumn{10}{c}{ Días observación } \\
\cline { 2 - 10 } & 1 & 2 & 3 & 4 & 5 & 6 & 7 & 8 & 9 \\
\hline Jacinto & $2.73 \mathrm{~b}$ & $2.60 \mathrm{~b}$ & $2.69 \mathrm{~b}$ & $3.02 \mathrm{~b}$ & $2.94 \mathrm{~b}$ & $3.16 \mathrm{~b}$ & $3.19 \mathrm{~b}$ & $3.53 \mathrm{~b}$ & $5.08 \mathrm{~b}$ \\
Lechuga & $2.75 \mathrm{a}$ & $1.51 \mathrm{a}$ & $1.72 \mathrm{a}$ & $2.07 \mathrm{a}$ & $1.80 \mathrm{a}$ & $1.64 \mathrm{a}$ & $1.88 \mathrm{a}$ & $2.12 \mathrm{a}$ & $2.68 \mathrm{a}$
\end{tabular}

Media con igual letra entre especies son no significativo al $p>0.05$.

El tratamiento SB mostró diferencia significativa ( $p$ $<0.05$ ) en todo el periodo de observación, a excepción del sexto día. SB presenta valores de 2.7 a $4.85 \mathrm{mg} \mathrm{l}^{-1} \mathrm{OD}$, superior al tratamiento CB de 1.33 a $2.91 \mathrm{mg} \mathrm{l}^{-1} \mathrm{OD}$ (Cuadro 2). Reddy et al. (1989) en un estudio similar en aguas residuales de origen doméstico de efluente primario, registró al final del octavo día valores de $1.1 \mathrm{mg} \mathrm{l}^{-1} \mathrm{OD}$ (Con Barrera) y $2 \mathrm{mg} \mathrm{l}^{-1}$ OD (Sin Barrera) utilizando plantas de lechugas, así mismo para jacinto reporta valores de $3.4 \mathrm{mg} \mathrm{l}^{-1} \mathrm{OD}(\mathrm{CB})$ y $4.9 \mathrm{mg}$ $1^{-1} \mathrm{OD}(\mathrm{SB})$. De acuerdo con los resultados obtenido en esta investigación, se confirma que el tratamiento SB presenta las mayores concentraciones de OD.

Cuadro 2. Oxígeno disuelto ( $\mathrm{mg} \mathrm{l}^{-1}$ ) en aguas residuales de origen acuícola con plantas con y sin barrera según días observados

\begin{tabular}{lccccccccc}
\hline \multirow{2}{*}{ Tratamiento 0} & \multicolumn{8}{c}{ Días de observaciones } \\
\cline { 2 - 10 } & 1 & 2 & 3 & 4 & 5 & 6 & 7 & 8 & 9 \\
\hline Plantas SB & $3.15 \mathrm{~b}$ & $2.70 \mathrm{~b}$ & $2.95 \mathrm{~b}$ & $3.20 \mathrm{~b}$ & $2.90 \mathrm{~b}$ & $2.73 \mathrm{a}$ & $3.16 \mathrm{~b}$ & $3.75 \mathrm{~b}$ & $4.85 \mathrm{~b}$ \\
Plantas CB & $1.33 \mathrm{a}$ & $1.42 \mathrm{a}$ & $1.46 \mathrm{a}$ & $1.89 \mathrm{a}$ & $1.85 \mathrm{a}$ & $2.06 \mathrm{a}$ & $1.90 \mathrm{a}$ & $1.89 \mathrm{a}$ & $2.91 \mathrm{a}$
\end{tabular}

$\overline{\text { SB: sin barrera, CB: con Barrera, media con similar letra en los tratamientos no son significativo } p}>0.05$.

Con referencia a la velocidad de transporte de OD, la especie de jacinto con y sin barrera presenta valores de 0.46 y $0.45 \mathrm{mg} \mathrm{O}_{2} \mathrm{~d}^{-1} \mathrm{l}^{-1}$ respectivamente (Cuadro 3), no muestra tendencia de aumento de concentraciones de OD y no establece un coeficiente de transferencia de OD al agua residual en el periodo observado. Estos datos están por debajo a lo reportado por Domínguez (2003), quien obtuvo en tres experimentos velocidades de transporte de 6.6, 7.3 y $5.2 \mathrm{mg} \mathrm{O}_{2} \mathrm{~d}^{-1} \mathrm{l}^{-1}$ utilizando planta de jacinto en un periodo de observación de nueve días. 
Cuadro 3. Oxígeno transportado en aguas residuales de origen acuícola utilizando planta de jacinto con y sin barrera

\begin{tabular}{|c|c|c|c|c|c|}
\hline \multirow{3}{*}{ Tratamiento } & \multicolumn{5}{|c|}{$\mathrm{O}_{2}$ transportado $=\mathrm{DBO}$ (inicial-final) $+\mathrm{OD}($ final-inicial $) / 9$ días } \\
\hline & \multicolumn{2}{|c|}{$\mathrm{DBO}_{5}$} & \multicolumn{2}{|c|}{ OD } & \multirow{2}{*}{$\begin{array}{c}\mathrm{O}_{2} \\
\text { Transportado }\end{array}$} \\
\hline & Inicial & Final & Inicial & Final & \\
\hline Jacinto SB & 21.40 & 17.06 & 5.63 & 5.91 & 0.45 \\
\hline Jacinto CB & 21.40 & 17.40 & 6.03 & 5.91 & 0.46 \\
\hline
\end{tabular}

SB: sin barrera; CB: con barrera; DBO: demanda biológica de oxígeno, OD: oxígeno disuelto.

La demanda biológica de oxígeno (DBO), en aguas residuales de origen acuícola con planta de jacinto $\mathrm{SB}$, presenta al inicio del experimento $21.4 \mathrm{mg} \mathrm{l}^{-1} \mathrm{y}$ al final del periodo redujo hasta $17.0 \mathrm{mg} \mathrm{l}^{-1}$, para una remoción del $20.60 \%$, mientras CB el porcentaje de remoción fue de 18.69 $\%$ muy por debajo a lo reportado por Reddy et al. (1989) en estudio similar de un sistema de tratamiento de agua domiciliar de un efluente primario con una DBO inicial 112.2 mg l-1$^{-1}$ utilizando jacinto removió el $73.9 \%$ DBO CB y SB $79.8 \%$. Se confirma en nuestro estudio que el tratamiento SB presenta mayor remoción de la DBO (Cuadro 4).

Cuadro 4. Remoción (\%) de DBO en aguas residuales de origen acuícola con jacinto sin y con barrera

\begin{tabular}{cccccc}
\hline \multirow{2}{*}{ Experimento } & \multirow{2}{*}{ Tratamiento } & \multicolumn{2}{c}{$\mathrm{DBO}\left(\mathrm{mg} \mathrm{l}^{-1}\right)$} & \multirow{2}{*}{ Diferencia } & \multirow{2}{*}{ \% Remoción } \\
\cline { 3 - 5 } & & Inicial & Final & & \\
\hline 1 & $\mathrm{CPSB}$ & 21.40 & 17.06 & 4.40 & 20.28 \\
1 & $\mathrm{CPCB}$ & 21.40 & 17.40 & 4.00 & 18.69 \\
\hline
\end{tabular}

CPSB: con plantas sin barrera, CPCB: con plantas con barrera.

DBO: Demanda biológica de oxígeno.

En tres experimentos con el tratamiento SPSB se registró remoción de $73 \%$, $33 \%$ y $85 \%$ DBO en aguas residuales de origen acuícola en un periodo de nueve días, mientras el SPCB remueve el $42 \%$. Estos resultados indican que el tratamiento SPSB está contribuyendo a la respiración de los microorganismos y su interacción con el ambiente. El tratamiento SPCB presenta los menores porcentajes de remoción de DBO ejercida por las actividades de las bacterias anaeróbicas (Cuadro 5).

Cuadro 5. Remoción (\%) de DBO sin planta sin o con barreras en aguas residuales de origen acuícola

\begin{tabular}{llcccc}
\hline \multirow{2}{*}{ Experimento } & \multirow{2}{*}{ Tratamiento } & \multicolumn{2}{c}{ DBO $\left(\mathrm{mg} \mathrm{l}^{-1}\right)$} & \multirow{2}{*}{ Diferencia } & $\%$ Remoción \\
\cline { 3 - 4 } & & Inicial & Final & & \\
\hline 1 & SPSB & 26.27 & 7.08 & 19.19 & 73.00 \\
2 & SPSB & 11.83 & 7.93 & 3.90 & 33.00 \\
3 & SPSB & 21.40 & 4.03 & 17.37 & 81.00 \\
Promedio & & & & & 63.33 \\
1 & SPCB & 26.27 & 15.29 & 10.98 & 42.00 \\
2 & SPCB & 11.83 & 16.10 & $4.27(-)$ & Sin remoción \\
3 & SPCB & 21.40 & 12.40 & 9.00 & 42.00 \\
Promedio & & & & & 42.00
\end{tabular}

SPSB: sin planta sin barrera, SPCB: sin planta con barrera, DBO: demanda biológica de oxígeno.
La biomasa seca de la planta de jacinto mostró mayor promedio de peso total seco (6.06 g) que lechuga de agua (3.20 g). Con la relación biomasa seca aérea - raíz (T/R), la planta de jacinto presentó un valor promedio de 0.62 lo que indica mayor peso de biomasa de la raíz comparada con biomasa aérea. Por el contrario, la planta de lechuga presentó mayor biomasa aérea con relación a la raíz, con un valor medio de 5.04 T/R (Cuadro 6). Reddy et al. (1989) encontró menor peso total promedio $(0.71 \mathrm{~g})$ y mayor $\mathrm{T} / \mathrm{R}(4.42)$ para jacinto, similar comportamiento presenta lechuga con peso total de $(1.49 \mathrm{~g})$ y T/R de 6.40. En el Cuadro 6, se observa la mayor concentración de OD en planta de jacinto con mayor peso de la biomasa total. Moorhead y Reddy (1988) reportan que en planta de jacinto existe alta correlación entre la biomasa de la raíz y oxígeno transportado, lo que representa que entre más pequeña es la biomasa de la raíz, mayor transporte de $\mathrm{O} 2$ del tejido aéreo hacia la rizosfera.

Cuadro 6. Biomasa seca de plantas macrophytas y contenido de oxígeno disuelto

\begin{tabular}{lccccc}
\hline \multicolumn{7}{c}{ Macrophytas } & Raíz & Tallo & $\begin{array}{c}\text { Peso } \\
\text { Total }\end{array}$ & $\begin{array}{c}\text { Relación } \\
\text { T/R }\end{array}$ & $\begin{array}{c}\text { OD } \\
\mathrm{mg} \mathrm{l}^{-1}\end{array}$ \\
\hline Jacinto SB & 4.85 & 2.23 & 7.08 & 0.46 & $3.63-5.34$ \\
Jacinto CB & 2.62 & 2.41 & 5.03 & 0.92 & $1.42-4.35$ \\
Promedio & 3.74 & 2.32 & 6.06 & 0.62 & \\
Lechuga SB & 0.61 & 2.40 & 3.01 & 3.93 & $1.7-4.81$ \\
Lechuga CB & 0.44 & 2.94 & 3.38 & 6.68 & $1.01-1.60$ \\
Promedio & 0.53 & 2.67 & 3.20 & 5.04 & \\
\hline
\end{tabular}

T/R: Relación tallo/raíz, OD: oxígeno disuelto, SB: sin barrera, $\mathrm{CB}$ : con barrera.

\section{CONCLUSIONES}

Existe una diferencia significativa $(p<0.05)$ entre especies de plantas acuáticas y tratamientos sin barrera y con barrera en cuanto a la concentración de oxígeno disuelto en aguas residuales en el periodo de nueve días de observación.

La planta de jacinto registra el mayor contenido de oxígeno disuelto (3.64 a $\left.5.37 \mathrm{mg}^{-1}\right)$ con referencia a lechuga de agua (1.51 a $2.75 \mathrm{mg} \mathrm{l}^{1-1}$ ), así mismo el tratamiento plantas sin barrera, registró mayores concentraciones de oxígeno disuelto $\left(2.7 \mathrm{a}^{2} .85 \mathrm{mg} \mathrm{l}^{-1}\right)$, comparado con barrera (1.33 a $\left.2.91 \mathrm{mg} \mathrm{l}^{-1}\right)$.

Jacinto reporta baja transferencia de oxígeno disuelto en aguas residuales con valores de 0.45 y $0.46 \mathrm{mg} \mathrm{O}_{2}$ $\mathrm{d}^{-1} \mathrm{l}^{-1}$ con barrera y sin barrera respectivamente, no muestra un coeficiente de transferencia de oxígeno disuelto.

Con relación a la remoción de la DBO en aguas residuales de origen acuícola con planta de jacinto sin barrera, presenta una remoción del $20.60 \%$, mientras con barrera fue de $18.69 \%$.

La remoción de la $\mathrm{DBO}$ en aguas residuales con tratamiento sin planta sin barrera (condiciones aeróbicas) fue 
del $62 \%$, mientras en condiciones anaeróbica (sin planta con barrera) obtuvo un valor de $42 \%$ en un periodo de observación de nueve días.

En la relación biomasa seca aérea/raíz (T/R), la planta de jacinto presentó un valor promedio de 0.62 lo que indica mayor peso de biomasa de la raíz comparada con biomasa aérea, así mismo mayor contenido de oxígeno disuelto de 3.63 a $5.34 \mathrm{mg}^{-1}$. Por el contrario, la planta de lechuga presenta mayor biomasa aérea con relación a la raíz con un valor medio de 5.04. La planta de jacinto mostró mayor peso total de biomasa seca de $6.06 \mathrm{~g}$ y concentración oxígeno disuelto de 1.42 a $5.34 \mathrm{mg} \mathrm{l}^{-1}$ en aguas residuales en comparación con lechuga de agua con un peso total de su biomasa de $3.20 \mathrm{~g}$ y OD de 1.01 a $4.81 \mathrm{mg} \mathrm{l}^{-1}$.

\section{REFERENCIAS BIBLIOGRÁFICAS}

Arriaza, V., Stanley Cáceres, M., Dueñas, A. L., Gil Laroj, J., Salazar Ortiz, C. y Rosales Escalante, E. (2004). Guía para el manejo de excretas y aguas residuales municipales. PROARCA/SIGMA.

Domínguez O., A. (2003). Transporte de oxígeno a través de plantas acuáticas. Scientia Et Technica, 1(21). https://revistas.utp.edu.co/ index.php/revistaciencia/article/view/7529

Fernández, J. (2004). Humedales artificiales para depuración. En J. Fernández González (coord.), Manual de fitodepuración:filtros de macrófitas en flotación[versión PDF]. España: EDITA. https://www.fundacionglobalnature.org/macrophytes/documentacion/ Cap\%edtulos\%20Manual/Cap\%edtulos\%206.pdf

Moorhead, K. K., y Reddy, K. R. (1988). Oxygen transport through selected aquatic macrophytes. American Society of Agronomy, Crop Science Society of America, and Soil Science Society of America 17(1), 138-142. https://soils.ifas.ufl.edu/wetlands/publications/ PDF-articles/129.Oxygen\%20transport\%20through\%20selected\%20aquatic\%20macrophytes..pdf

Reddy, K. R., D'angelo, E. M., y DeBusk, T. A. (1989). Oxygen transport through aquatic macrophytes: the role in wastewater treatment. J. Environ. Qual 19(2), 261-267. https://www.oieau.fr/eaudoc/system/files/documents/3/15550/15550_doc.pdf

Schutze, C. E. (2010). Experiencias en saneamiento integral en Nicaragua 